\title{
EDITORIAL: TECHNOLOGY AND THE POSTINVESTMENT FAILURE
}

The rapid development and spread of medical technology in the last thirty years has changed health care dramatically and forced authorities in most countries to consider the effects of high-technology medicine and the use of sophisticated equipment within health care. Medical technology tends to institutionalize health care, creates sophisticated organizational and administrative systems, and because of the size of the investment involved, competes for money with other broadly directed health care actions.

\section{POSTINVESTMENT FAILURE}

The postinvestment failure in technology is the failure to reach the expected payoff: of investment in it because either the investment was not appropriate for the needs, purpose, culture, educational level, or economy of the country, or it was not used appropriately by the country's medical care system, which might lack, for example, an adequate means of technological maintenance.

Postinvestment failure is by no means a problem of developing countries alone. It is a worldwide phenomenon, reflecting the accelerating expansion of technology and the fast dissemination of health technology in relation to the much slower development of social structure, general knowledge, and cultural and ethical value constancy. Postinvestment failure should therefore be dealt with from many different angles.

\section{THE PREVENTION OF THE POSTINVESTMENT fAILURE}

The prevention of postinvestment failure requires health care policymakers to address a series of questions before procurement and introduction of a medical technology are made. Such questions are: Is the technology appropriate for the country's expressed needs? Does it deal with an important health problem? Will it change the outcome for patients suffering from that health problem? Is it, for instance, reasonable to introduce a new, sophisticated, expensive diagnostic technology that diagnoses an illness more accurately than was done previously, even if there is no effective treatment for that condition or if the new method cannot exclude a disease that otherwise would likely have been wrongly diagnosed and treated? Is the new technology economically acceptable, or does it divert too many resources towards diagnoses and treatments which may not have large epidemiological importance or provide only minor gains in survival or life quality? (Some transplant programs are examples of that.) Is the technology culturally appropriate? For example, it would hardly be meaningful to introduce amniocentesis in countries where the culture does not allow abortion. Also, are there alternative technologies which are cheaper, though not as fully effective? If the cost is substantially lower, such a method may be more cost-effective. Thus, it 
may be feasible to offer treatment to larger groups of patients, and the health benefit for society will increase despite a lower efficacy of the method. For example, method A cures $95 \%$ of the target population at a cost of $\$ 100,000$; method $B$ cures $75 \%$ of the target population at a cost of $\$ 10,000$. Is the method acceptable from a medical point of view, and of course is it acceptable from that of the patient? All these questions of assessment should be asked before the introduction and spread of a technology.

\section{LEGISLATION, PLANNING, RESOURCE ALLOCATION, MANAGERIAL PROCESS}

One major dimension of the postinvestment failure is the social structure and decision-making process in a country and its ability to approach the postinvestment phase in a rational way. Very often major maintenance and repair problems are to be found not at the site of the apparatus, but in the ministry of health or finance, or with export/import or customs authorities. To enjoy the benefits of high-technology hardware it is not enough to have access to it. It is also necessary to have a decision-making system that is adapted to high technology. This often means a high degree of flexibility and decentralization of decisions, and less detailoriented administrative rules and regulations. By and large, the development of a flexible and rapid decision-making system seems to be a prerequisite for the optimal functioning of high technology.

Managerial ineffectiveness is a problem all over the world, partly because of lack of research, managerial know-how, and funds. It is therefore interesting to note that managerial support is now being marketed in some countries. Until recently, manufacturers of medical products saw their role primarily as the research and development, manufacturing, marketing, and delivery of quality products. This is now changing, and several companies are expanding their role from the mere production of medical products to the delivery of services, including managerial support. In many hospitals, products and materials management represent nearly $40 \%$ of the hospital budget. The introduction of managerial support systems that permit a more rational procurement and handling of all types of equipment now seems important. It is crucial to develop and strengthen the current managerial processes for health development at large, with this having special relevance to the procurement, use, and maintenance of advanced medical technology.

\section{CONCLUSIONS}

Since investment in health technology is competing with that in other health areas, as well as with investment in the social or educational development of a country, administrators must carefully consider which technology is appropriate for the defined need of that country through a formal assessment process. Care should be taken to maintain the value of the investment by means of effective legislation, flexible rules and regulations, and competent managerial processes. The appropriate use of technologies should be guaranteed through constant evaluation and quality assurance, the result of which should create data suited for analyses useful for identifying priorities and for policymaking. This should be the responsibility 
of all levels of the health care system from policymakers to appliers. Therefore, education for policymakers, providers, and patients is necessary. Equipment maintenance should be promoted, and only such technology as can be expected to conserve its usefulness long enough to be cost-effective should be introduced.

Johannes Vang 


\title{
AN INTERNATIONAL VIEW OF MAGNETIC RESONANCE- IMAGING AND SPECTROSCOPY
}

\section{Guest Editors}

\author{
Tore Scherstén, M.D. \\ Dean, Faculty of Medicine \\ Department of Surgery \\ University of Göteborg \\ Jane E. Sisk, Ph.D. \\ Health Program \\ Office of Technology Assessment \\ United States Congress
}

